Ghislaine Douflé Francesca Facchin Shabana Anwar Eddy Fan

\section{Severe hypercoagulable state on veno-arterial extracorporeal membrane oxygenation}

Received: 22 August 2015

Accepted: 27 August 2015

Published online: 11 September 2015

(C) Springer-Verlag Berlin Heidelberg and ESICM 2015

G. Douflé and F. Facchin contributed equally to this work.

Electronic supplementary material The online version of this article (doi:10.1007/s00134-015-4048-y) contains supplementary material, which is available to authorized users.

G. Douflé · F. Facchin · S. Anwar · E. Fan

Interdepartmental Division of Critical Care Medicine,

University of Toronto, Toronto, Canada

G. Douflé $(\bullet) \cdot$ F. Facchin · S. Anwar · E. Fan

Extracorporeal Life Support (ECLS) Program, Toronto General Hospital, 585 University Ave, PMB 11-123, Toronto, ON M5G 2N2, Canada

e-mail: ghis.doufle@gmail.com; ghislaine.doufle@uhn.ca ghislaine.doufle@mail.utoronto.ca

Tel.: (416) 340-4800

A previously healthy 49 -year-old female presented to the emergency department with chest pain and was diagnosed with antero-lateral ST-segment elevation myocardial infarction. Urgent coronary angiography demonstrated the presence of transient coronary thrombi in the left anterior descending and circumflex arteries with no obvious plaque rupture. Severe biventricular dysfunction was also noted.

During the angiogram, she became extremely hemodynamically unstable. She was intubated and the decision was made to initiate hemodynamic support with peripheral veno-arterial extracorporeal membrane oxygenation (ECMO). Concurrently, acute thrombi had formed in both

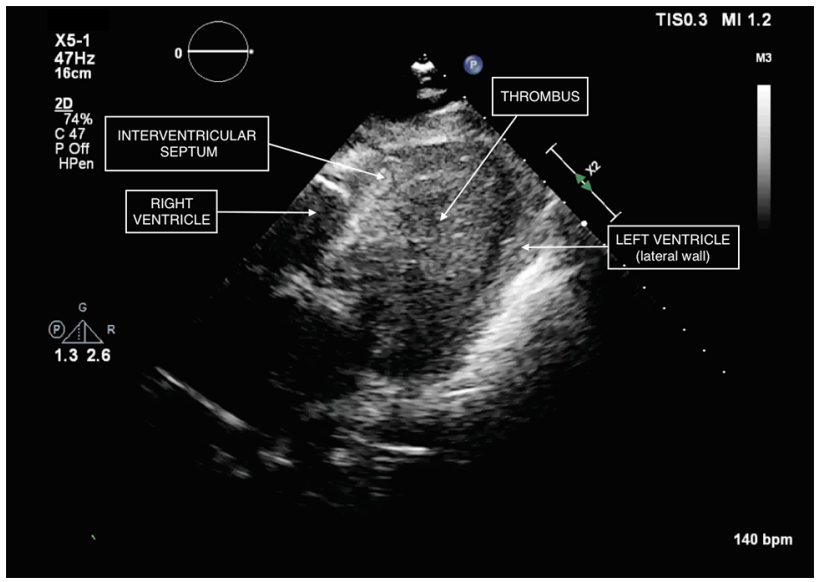

Fig. 1 Transthoracic echocardiography. Subcostal view with massive thrombus filling the left ventricular cavity

iliac arteries during the procedure. Initial echocardiography showed a moderate pericardial effusion without signs of tamponade, a virtually non-contracting heart and a closed aortic valve.

Due to severe coagulopathy, anticoagulation (normally commenced for ECMO) was withheld. On ICU day 2, abrupt hemodynamic deterioration with massive pulmonary edema prompted a repeat echocardiography, which demonstrated a massive thrombus filling the entire left ventricle (Fig. 1; Video 1). The presence of severe concomitant thrombosis and bleeding was deemed to be an inextricable situation, and after discussion with the patient's next of kin, life support was discontinued.

Compliance with ethical standards

Conflicts of interest On behalf of all authors, the corresponding author states that there is no conflict of interest. 